\title{
Ventilación no invasiva en pacientes con enfermedad pulmonar obstructiva crónica e insuficiencia respiratoria aguda hipercápnica en una sala de hospitalización convencional
}

\author{
F. J. GONZÁLEZ BARCALA, C. ZAMARRÓN SANZ, M. SALGUEIRO \\ RODRÍGUEZ, J. R. RODRÍGUEZ SUÁREZ
}

Servicio de Neumología. Hospital Clínico Universitario. Santiago de Compostela

\begin{abstract}
NON-INVASIVE VENTILATION IN CHRONIC OBSTRUCTIVE PULMONARY DISEASE PATIENTS WITH ACUTE RESPIRATORY HYPERCAPNIC FAILURE IN A CONVENTIONAL HOSPITAL WARD
\end{abstract}

\begin{abstract}
RESUMEN
Objetivo: Valorar el tratamiento con ventilación mecánica no invasiva (VNI) de pacientes con enfermedad pulmonar obstructiva crónica (EPOC) e insuficiencia respiratoria aguda hipercápnica en una planta de hospitalización.

Método: Estudio prospectivo de 35 pacientes con EPOC agudizada que fueron tratados con ventilación con presión positiva intermitente mediante mascarilla oro-nasal en una sala de hospitalización convencional. En situación estable, los pacientes presentaban una relación FEV1/FVC del 55,3 $\pm 14,8 \%$ del teórico y $17(48,5 \%)$ seguían oxigenoterapia continua domiciliaria. Hemos analizado los datos gasométricos basalmente y a diferentes periodos del inicio de la VNI, así como las complicaciones y fracasos de este tratamiento.

Resultados: Se observa una mejoría significativa de los gases sanguíneos tras 24 horas de VNI con respecto a los niveles básales. Los pacientes presentaron una estancia hospitalaria media de 15,1 $\pm 9,1$ días. 13 pacientes $(37 \%)$ presentaron escaras faciales y 3 pacientes $(8,5 \%)$ fallecieron.

Conclusiones: La VNI en una planta de hospitalización es un medio terapéutico útil y seguro para los pacientes en insuficiencia respiratoria aguda hipercápnica tras una agudización de EPOC.
\end{abstract}

PALABRAS CLAVE: Ventilación mecánica. Presión positiva intermitente. Enfermedad pulmonar obstructiva crónica. Insuficiencia respiratoria. Acidosis respiratoria.

\begin{abstract}
Objective: To analyze chronic obstructive lung disease (COPD) subjects in acute hypercapnic failure who were treated with non-invasive mechanical ventilation in a general respiratory ward.

Methods: This was a two-year prospective study of 35 patients with acute exacerbation of COPD and mean FEVI/FVC relation in stable condition of $55.3 \pm 14.8 \%$ of predicted that were treated with positive pressure respiration using a facemask in a general respiratory ward. 17 $(48,5 \%)$ receive long-term oxygen therapy. Analysis was made of blood gases, before and after treatment of non-invasive ventilation, complications, and failure during treatment.

Results: A significant improvement in blood gases was observed 24 hours after non-invasive ventilation treatment. The mean hospital stay was of $15.0 \pm 9.1$ days and failures were registered in 3 cases $(8.5 \%)$. Facial scares were the most common complication (13 patients) but it was possible to continue treatment.

Conclusions: Non-invasive ventilation is a viable treatment for patients with chronic obstructive lung disease and acute hypercapnic failure being treated in a general respiratory ward.
\end{abstract}

KEY WORDS: Mechanical Ventilation. Positive Pressure Respiration. Chronic Obstructive Airway Disease. Respiratory Insufficiency. Acidosis respiratory.

González Barcala FJ, Zamarrón Sanz, C, Salgueiro Rodríguez, M, Rodríguez Suárez JR. Ventilación no invasiva en pacientes con enfermedad pulmonar obstructiva crónica e insuficiencia respiratoria aguda hipercápnica en una sala de hospitalización convencional. An Med Interna (Madrid) 2004; 21: 373-377.

\section{INTRODUCCIÓN}

La enfermedad pulmonar obstructiva crónica (EPOC) es un problema sanitario de primera magnitud debido a su elevada prevalencia y a la alta utilización de recursos sanitarios que lleva consigo siendo una de las causas principales de consulta médica (1). La EPOC puede presentar durante su evolución episodios de reagudización e insuficiencia respiratoria aguda que requiere, junto a un tratamiento farmacológico más intenso y a la oxigenoterapia, la intubación traqueal y la ventilación mecánica. La necesidad de mejorar el tratamiento de estos pacientes durante la reagudización, evitando las compli- caciones asociadas a la intubación traqueal y optimizando la estancia hospitalaria han facilitado la inclusión de la ventilación no invasiva (VNI), como una modalidad terapéutica del fracaso ventilatorio (2-7). Sin embargo, existe discusión acerca de los criterios de indicación, así como el lugar de realización de esta modalidad terapéutica $(4,5,7-9)$, dado que la mayoría de estos estudios han sido realizados en Cuidados Intensivos (2,10-12) y estos resultados pueden no ser trasladables a otras ubicaciones hospitalarias.

El objetivo de nuestro estudio fue valorar el tratamiento con VNI pacientes con EPOC e insuficiencia respiratoria aguda hipercápnica en una planta de hospitalización convencional.

Trabajo aceptado: 18 de marzo de 2004

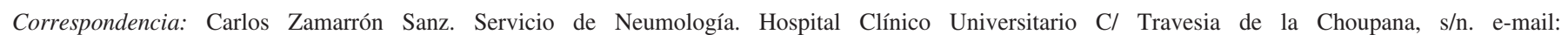
carlos.zamarron.sanz@sergas.es.15706 Santiago de Compostela 


\section{MATERIAL Y MÉTODO}

Desde enero de 2000 hasta diciembre de 2001, ambos inclusive hemos estudiado 35 pacientes con EPOC que acudieron al hospital Urgencias con insuficiencia respiratoria hipercápnica aguda y que tras su valoración por los Servicios de Urgencias, Neumología y Cuidados Intensivos eran ingresados en la planta de hospitalización de Neumología (13). 13 pacientes $(37 \%)$ eran EPOC graves y $22(63 \%)$ EPOC moderados (6). Las características clínicas y funcionales de estos pacientes se presentan en la tabla I.

\section{TABLA}

\section{CARACTERÍSTICAS CLÍNICAS Y FUNCIONALES DE LOS} PACIENTES

\begin{tabular}{|c|c|}
\hline Variable & \\
\hline Varón / mujer, n & $30 / 5$ \\
\hline Edad, años & $71,7 \pm 9,9(49-88)$ \\
\hline $\mathrm{IMC}, \mathrm{kg} / \mathrm{m}^{2}$ & $31,6 \pm 6,4(20-45)$ \\
\hline Estancia hospitalaria (días) & $15,0 \pm 9,1(3-55)$ \\
\hline OCD $(\%)$ & $17(48,5 \%)$ \\
\hline $\mathrm{pH}$ basal & $7,29 \pm 0,05(7,19-7,35)$ \\
\hline PO2 basal (mmHg) & $46,8 \pm 8,79(32-67)$ \\
\hline PCO2 basal (mmHg) & $75,4 \pm 13,8(52-106)$ \\
\hline Bicarbonato basal (mEq/l) & $35,6 \pm 5,1(23-45)$ \\
\hline $\mathrm{FEV}_{1} \mathrm{I} / \mathrm{s}$ & $1,2 \pm 0,5(0,5-2,2)$ \\
\hline FEV 1 teórico $(\%)$ & $48,7 \pm 1,2$ \\
\hline FVC (I) & $2,5 \pm 1,1(1,0-4,8)$ \\
\hline FVC teórico (\%) & $59,9 \pm 16,2$ \\
\hline $\mathrm{FEV}_{1} / \mathrm{FVC}(\%)$ & $52,5 \pm 13,1(26-70)$ \\
\hline
\end{tabular}

OCD: Oxigenoterapia continua domiciliaria, $\mathrm{PO}_{2}$ basal $: \mathrm{PO}_{2}$ antes del inicio del tratamiento con $\mathrm{VNI} ; \mathrm{PCO}_{2}$ basal $: \mathrm{PCO}_{2}$ antes del inicio del tratamiento con VNI; PH basal: $\mathrm{pH}$ antes del inicio del tratamiento con VNI.

Como técnica de VNI se ha utilizado la presión de soporte aportada por un respirador de presión positiva intermitente (Respironic ${ }^{\circledR}$, Atlanta USA) en modo espontáneotiempo, de forma continuada durante las primeras 24-48 horas, seguido de forma discontinua durante 2 horas por la mañana y tarde y toda noche hasta conseguir la mejoría clínica y normalización del $\mathrm{pH}$ sanguíneo. Los parámetros ventilatorios utilizados dependían de la tolerancia y adaptación del paciente. Se iniciaba el tratamiento con una presión positiva inspiratoria (IPAP) de $12 \mathrm{~cm}$ de $\mathrm{H}_{2} \mathrm{O}$ y una presión espiratoria (EPAP) de $4 \mathrm{~cm}$ de $\mathrm{H}_{2} \mathrm{O}$, aportando oxígeno suplementario para mantener $\mathrm{SaO}_{2}$ entre el 85-90\%. Se realiza una gasometría arterial a los 60 minutos de estos primeros ajustes. La presión de IPAP se incrementa progresivamente hasta niveles entre 18 y $22 \mathrm{~cm}$ de $\mathrm{H}_{2} \mathrm{O}$ y la EPAP hasta 6 a $10 \mathrm{~cm}$ de $\mathrm{H}_{2} \mathrm{O}$, en función de la evolución clínicogasométrica del paciente. La frecuencia respiratoria inicial programada fue 2 puntos por debajo de la espontánea, hasta lograr el acoplamiento del paciente al ventilador y la relación de tiempos inspiratorio/espiratorio fue de $1 / 3$. Se utilizó una mascarilla oronasal con un tamaño adecuado en cada paciente (Fig. 1).

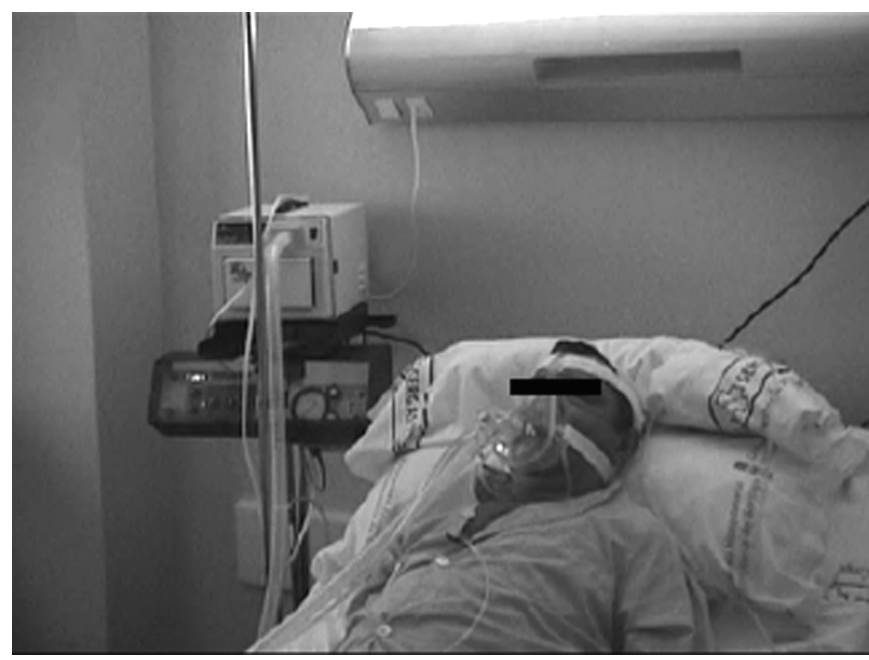

Fig. 1. Paciente en tratamiento con ventilación positiva intermitente a través de un mascara oronasal.

Cada paciente mantenía el tratamiento medicamentoso necesario consistente en salbutamol, bromuro de ipratropio, teofilina, corticoides sistémicos y antibióticos o diuréticos si se requerían. Se realiza gasometría basal y controles gasométricos a la hora, 6 horas y 24 horas de iniciada la ventilación, así como pulsioximetría continua; realizándose posteriormente según evolución clínica al menos cada 24 horas hasta el alta. Se suspendió la VNI cuando se producía la normalización de los parámetros gasométricos ( $\mathrm{pH}$ mayor de 7,35 y $\mathrm{pCO}_{2}$ menor o igual de $45 \mathrm{mmHg}$ ) o desaparición de la acidosis con persistencia de la hipercapnia que se mantenía estable y era la habitual en ese paciente durante las fases estables. Se considera fracaso de la técnica el rechazo del paciente tras inicio de la misma, la mala respuesta clínica o gasométrica que requiera intubación endotraqueal, el traslado a Cuidados Intensivos o el éxitus.

En la planta de hospitalización no se ha modificado la dotación de personal de enfermería durante el ingreso de estos pacientes. El seguimiento de enfermería del tratamiento se centra en la vigilancia de la tolerancia del paciente, posibles fugas o desconexiones del equipo, saturación de oxígeno, además de los cuidados propios de un paciente EPOC agudizado grave. Se permitió la estancia de un familiar con el paciente de forma continuada.

\section{ANÁLISIS ESTADÍSTICO}

Las variables continuas son expresadas como media y como coeficiente de dispersión se utiliza la desviación estándar. Se utiliza la t de Student para comparación de medias con distribución normal y la U de Mann Whitney para aquellas con distribución no paramétrica. Se realizaron comparaciones entre los datos del intercambio gaseoso de las diferentes muestras utilizando el test de Friedman. Asimismo, se compararon los datos del intercambio gaseoso en ventilación espontánea con los obtenidos a la hora, 6 horas y 24 horas del inicio del soporte ventilatorio así como al alta, aplicando el test de Wilcoxon con el ajuste de Dunnet. Las variables categóricas se expresan en porcentajes, utilizando la prueba de Chi cuadrado para la comparación de 
TABLA II

EVOLUCIÓN GASOMÉTRICA DE LOS PACIENTES

\begin{tabular}{lccccc}
\hline & Basal & 1 hora & 6 horas & 24 horas & Alta \\
\hline $\mathrm{PO}_{2}(\mathrm{mmHg})$ & $46,8 \pm 8,8$ & $77,2 \pm 51,4^{* *}$ & $77,6 \pm 28,3^{* *}$ & $86,9 \pm 44,6^{* *}$ & $69,1 \pm 20,7^{* *}$ \\
$\mathrm{PCO}_{2}(\mathrm{mmHg})$ & $75,4 \pm 13,8$ & $78,7 \pm 13,6$ & $73,7 \pm 13,4$ & $66,9+12,0^{*}$ & $53,5 \pm 12,6^{* *}$ \\
$\mathrm{pH}$ & $7,29 \pm 0,05$ & $7,28 \pm 0,06$ & $7,30 \pm 0,05$ & $7,37 \pm 0,05^{* *}$ & $7,42 \pm 0,04^{* *}$ \\
Bicarbonato $(\mathrm{mEq} / \mathrm{l})$ & $35,6 \pm 5,0$ & $36,3 \pm 5,4$ & $36,5 \pm 5,5$ & $37,5 \pm 5,2$ & $34,1 \pm 8,5$ \\
\hline
\end{tabular}

Basal: Antes del inicio del tratamiento con $\mathrm{VNI}$; 1 hora: A la hora del inicio del tratamiento con VNl; 6 horas: A las 6 horas de iniciado el tratamiento; 24 horas: A las 24 horas de iniciado el tratamiento; Alta: Gasometría al alta.

estas variables. Se considera significación estadística un valor de $\mathrm{p}<0,05$.

Para verificar la existencia de asociación entre las distintas variables clínicas y detectar aquellas con capacidad de predecir la respuesta al soporte ventilatorio a las 24 horas, se ha confeccionado un modelo de regresión logística siguiendo un proceso escalonado. Sólo las variables significativas $(\mathrm{p}<0,05)$, fueron incluidas en el modelo. Los riesgos relativos estimados y su intervalo de confianza al 95\% fueron calculados a partir de los coeficientes.

\section{RESULTADOS}

Los datos del intercambio gaseoso en situación basal y la evolución gasométrica tras el inicio del inicio del soporte ventilatorio se presentan en la tabla II. A partir de la primera hora se observa una mejoría de la $\mathrm{PO}_{2}$ con respecto a los niveles básales. Los niveles de $\mathrm{pCO}_{2}$ caen de forma significativa a las 24 horas, normalizándose también el pH (Tabla II).

Únicamente el $12 \%$ los pacientes redujeron más de un

\section{TABLA III}

CARACTERÍSTICAS CLÍNICAS Y FUNCIONALES DE LOS PACIENTES CON Y SIN OXIGENOTERAPIA CONTINUA DOMICILIARIA

\begin{tabular}{lccc}
\hline & $\mathrm{OCD}(\mathrm{n}=17)$ & $\operatorname{Sin} \mathrm{OCD}(\mathrm{n}=18)$ & $\mathrm{p}$ \\
\hline Estancia hospitalaria (días) & $15,2 \pm 12,7$ & $14,0 \pm 5,3$ & $\mathrm{NS}$ \\
Edad (años) & $74,1 \pm 7,5$ & $69,6 \pm 11,5$ & $\mathrm{NS}$ \\
$\mathrm{FEV} 1 / \mathrm{FVC}(\%)$ & $44,3 \pm 12,7$ & $66,5 \pm 13,7$ & 0,01 \\
$\mathrm{IMC}_{1} \mathrm{~kg} / \mathrm{m}^{2}$ & $28,7 \pm 6,7$ & $32,9 \pm 6,1$ & $\mathrm{NS}$ \\
$\mathrm{PO}_{2 \text { basal }}(\mathrm{mmHg})$ & $45,8 \pm 14,4$ & $44,6 \pm 8,6$ & $\mathrm{NS}$ \\
$\mathrm{PO}_{21 \text { hora }}(\mathrm{mmHg})$ & $85,3 \pm 13,6$ & $87,0 \pm 69,2$ & $\mathrm{NS}$ \\
$\mathrm{PO}_{2 \text { 6horas }}(\mathrm{mmHg})$ & $75,9 \pm 25,8$ & $79,1 \pm 30,8$ & $\mathrm{NS}$ \\
$\mathrm{PO}_{2 \text { 24 hora }}(\mathrm{mmHg})$ & $81,8 \pm 39,4$ & $80,3 \pm 37,2$ & $\mathrm{NS}$ \\
$\mathrm{PCO}_{2 \text { basal }}(\mathrm{mmHg})$ & $83,1 \pm 11,9$ & $68,6 \pm 11,8$ & 0,001 \\
$\mathrm{PCO}_{2 \text { h }}(\mathrm{mmHg})$ & $85,3 \pm 13,6$ & $72,8 \pm 10,9$ & 0,005 \\
$\mathrm{PCO}_{2 \text { hora }}(\mathrm{mmHg})$ & $78,0 \pm 13,9$ & $70,1 \pm 9,9$ & 0,05 \\
$\mathrm{PCO}_{2 \text { 24 horas }}(\mathrm{mmHg})$ & $68,5 \pm 11,2$ & $65,4 \pm 12,8$ & $\mathrm{NS}$ \\
$\mathrm{pH}_{\text {basal }}$ & $7,27 \pm 0,04$ & $7,30 \pm 0,05$ & $\mathrm{NS}$ \\
$\mathrm{pH}_{1 \text { hora }}$ & $7,28 \pm 0,05$ & $7,29 \pm 0,06$ & $\mathrm{NS}$ \\
$\mathrm{pH}_{6 \text { horas }}$ & $7,30 \pm 0,06$ & $7,31 \pm 0,05$ & $\mathrm{NS}$ \\
$\mathrm{pH}_{24 \text { horas }}$ & $7,38 \pm 0,05$ & $7,37 \pm 0,06$ & $\mathrm{NS}$ \\
\hline $\mathrm{OCD}_{\text {: Oxig }}$ & &
\end{tabular}

OCD: Oxigenoterapia continua domiciliaria; IMC: índice de masa corporal; $\mathrm{PO}_{2 \text { basal }} \mathrm{PO} 2$ antes del inicio del tratamiento con $\mathrm{VNI} ; \mathrm{PO}_{21 \text { hora }}: \mathrm{PO}_{2}$ a la hora del inicio del tratamiento; $\mathrm{PO}_{26 \text { horas }} \mathrm{PO}_{2}$ a las 6 horas; $\mathrm{PO}_{24}$ horas: $\mathrm{PO}_{2}$ a las 24 horas; $\mathrm{PCO}_{2 \text { basal }}: \mathrm{PCO}_{2}$ antes del inicio del tratamiento con $\mathrm{VNl} ; \mathrm{PCO}_{21}$ : $\mathrm{PCO}_{2}$ a la hora del inicio del tratamiento; $\mathrm{PCO}_{26 \text { hora }} \mathrm{S}$ : $\mathrm{PCO}_{2}$ a las 6 horas; $\mathrm{PCO}_{24 \text { horas }}: \mathrm{PCO}_{2}$ a las 24 horas; $\mathrm{PH}_{\text {basal }}$ : $\mathrm{pH}$ antes del inicio del tratamiento con $\mathrm{VNI} ; \mathrm{PH}_{1 \text { hora }}$ : $\mathrm{pH}$ a la hora del inicio del tratamiento: $\mathrm{PH}_{6 \text { horas }}$ : $\mathrm{pH}$ a las 6 horas; $\mathrm{PH}_{24 \text { horas }}$ : $\mathrm{pH}$ a las 24 horas.
TABLA IV

VARIABLES CLÍNICAS QUE HAN EVIDENCIADO LA CAPACIDAD DE PREDECIR LA RESPUESTA A LAS 24 HORAS AL TRATAMIENTO CON VNI

\begin{tabular}{lccccc}
\hline & Coeficiente $b$ & Error estándar & $R R$ & $I C$ al $95 \%$ & $p$ \\
\hline Edad & 0,076 & 0,040 & 1,10 & $1.00-1,17$ & 0,050 \\
OCD & -1.917 & 0,911 & 0,15 & $0,02-0,091$ & 0,035 \\
Constante & -1.599 & 3,613 & & & \\
\hline
\end{tabular}

OCD: Oxigenoterapia continua domiciliaria; RR: Riesgo relativo; IC al $95 \%$ = Intervalo de confianza al $95 \%$.

$10 \%$ la $\mathrm{pCO}_{2}$ tras una hora de soporte ventilatorio, $30 \%$ a las 6 horas y el 60\% a las 24 horas del inicio de la VNI.

Las características clínicas y funcionales de los pacientes con y sin oxígeno domiciliario se muestran en la tabla III. Diecisiete pacientes $(48,5 \%)$ seguían tratamiento con oxigenoterapia continua domiciliaria y $18(51,5 \%)$ no precisaban este tratamiento. No existen diferencias entre ambos grupos con respecto a la edad, IMC y estancia hospitalaria. Los pacientes con oxigenoterapia continua domiciliaria presentan una disminución significativa de la relación FEV1/FVC y un incremento de los valores de $\mathrm{pCO}_{2}$, tanto basalmente como a la hora y seis horas del inicio del soporte ventilatorio, sin diferencias en las cifras de $\mathrm{pO}_{2}$ o de $\mathrm{pH}$.

La respuesta al tratamiento con VNI a las 24 horas se asocia a la edad y al antecedente de seguir oxigenoterapia continua domiciliaria. Los pacientes con más edad y oxigenoterapia continua domiciliaria tienen peor respuesta al soporte ventilatorio a las 24 horas.

Tres enfermos presentaron aerofagia y 13 presentaron escaras faciales sin que fuera necesario retirar el soporte ventilatorio. Ha habido 3 fracasos (un éxitus en planta, un traslado a Cuidados Intensivos con éxitus en la misma y un éxitus tras mejoría inicial y rechazo por parte del paciente de la continuación del soporte ventilatorio), lo cual supone una mortalidad global del 8,5\%.

\section{DISCUSIÓN}

En el presente estudio la VNI, utilizada en la planta de hospitalización convencional, se mostró como una alternativa terapéutica útil en más del $80 \%$ de pacientes con insuficiencia respiratoria aguda hipercapnica asociada a la reagudización de la EPOC.

Durante los últimos años la VNI ha adquirido progresivamente mayor protagonismo en el tratamiento del fracaso ventilatorio agudo de los pacientes con EPOC debido a que redu- 
ce la mortalidad y complicaciones asociadas a la intubación orotraqueal (9), además de ser altamente costo-efectiva (14). Al aplicar este tratamiento en la planta de hospitalización surgen controversias sobre la utilidad del mismo, ante la discordancia de los resultados observados en la literatura, demostrándose beneficioso según unos autores, pero no siendo de utilidad según otros. Antón y cols. en un estudio prospectivo de 59 casos de agudización clínica en pacientes con una EPOC obtuvieron un $77 \%$ de éxitos y objetivaron que una mejoría del $\mathrm{pH}$, de la $\mathrm{PaCO}_{2}$ y del nivel de conciencia, tras la primera hora de ventilación, permitía predecir el éxito de la VNI en la mayoría de los casos (15). En un estudio multicéntrico realizado durante dos años en catorce hospitales, Plant y cols. objetivaron que la VNI reducía la frecuencia de las intubaciones orotraqueales y la mortalidad asociada (16). En cambio, en un estudio retrospectivo de 49 pacientes se comparó la respuesta gasométrica de un grupo de pacientes con EPOC agudizada y tratamiento convencional (broncodilatadores, antibióticos, glucocorticoides y oxigenoterapia) con la de otro al que se asociaba la VNI, no objetivándose ninguna ventaja en aquel que fue ventilado (17). En otro estudio prospectivo de 24 pacientes, 20 fueron distribuidos en dos grupos de 10 (4 no toleraron la mascarilla), uno con tratamiento convencional (oxigenoterapia, broncodilatadores y antibióticos) y el otro combinándolo con VNI. Las distintas variables analizadas, tales como el promedio de estancia hospitalaria, la oxigenación arterial, el equilibrio ácido-base y la función pulmonar mejoraron en los dos subgrupos sin diferencias significativas. Los autores concluyeron que la VNI no está indicada en el tratamiento convencional de las agudizaciones de la EPOC fuera de las unidades de Cuidados Intensivos (18).

La gravedad de la acidosis respiratoria $(16,19)$ y la edad (20) constituyen importantes factores pronósticos en pacientes con EPOC en insuficiencia respiratoria aguda. Las mayores tasas de intubación orotraqueal y de mortalidad corresponden a los pacientes con mayor nivel de acidosis. En el presente estudio hemos encontrado que la edad y el antecedente de estar sometido a oxigenoterapia continua domiciliaria están relacionados con la respuesta al soporte ventilatorio a las 24 horas.

Las agudizaciones de EPOC están asociadas a un 14\% de mortalidad (21) y al $32 \%$ cuando ingresan en Cuidados Intensivos (22). En nuestro estudio la mortalidad fue del 8,5\% y también es inferior a la aportada por otros grupos que utilizan la VNI como tratamiento de estos enfermos en la planta de hospitalización. En el estudio de Bott se contabiliza un 13\%

\section{Bibliografía}

1. Sobradillo V, Miravitlles M, Jiménez CA, Gabriel R, Viejo JL, Masa JF, Fernández-Fau L, Villasante C. Estudio IBERPOC en España: prevalencia de síntomas respiratorios habituales y de limitación crónica al flujo aéreo. Arch Bronconeumol 1999; 35: 159-66.

2. Brochard L, Mancebo J, Wysocki M, Lofaso F, Conti G, Rauss A, Simonneau G, Benito S, Gasparetto A, Lemaire F, Isabey D, Harf A. Noninvasive ventilation for acute exacerbations of chronic obstructive pulmonary disease. N Engl J Med 1995; 333: 817-822.

3. Keenan SP, Gregor J, Sibbald WJ, Cook D, Gafni A. Noninvasive posi- de fracasos, teniendo en cuenta que 4 pacientes randomizados al grupo de VNI, no reciben este tratamiento por diversos motivos (2 por bajo nivel de conciencia, uno no tolera y otro rechaza) (19). Confalonieri presenta resultados con un $17 \%$ de fracasos y un $12 \%$ de mortalidad, con un protocolo y ventiladores similares a los utilizados por nosotros pero en un grupo de menor edad, menor hipercapnia y similares acidosis y grado de obstrucción ( $\mathrm{pH}$ de 7,29, FEV1/FVC de 57\%) (23). Antón refiere un $9 \%$ de mortalidad. La población de este trabajo es de más edad que la nuestra, con más severidad en cuanto a acidosis y a hipercapnia (15). Bardi aplica la VNI durante al menos 20 horas durante el primer día, de forma similar a nuestro protocolo, en pacientes más jóvenes, con similar grado de obstrucción al flujo aéreo aunque con menos acidosis respiratoria. En el análisis de los resultados, excluye dos pacientes que no toleran la ventilación, que de ser incluidos elevaría la tasa de fracasos al 20\% (24). En un trabajo reciente se aplica VNI en la planta de hospitalización a pacientes con encefalopatía hipercápnica. Se trata de pacientes más graves que los incluidos en nuestro estudio, con una media de $\mathrm{pH}$ de 7,18 , presentando un $31 \%$ de mortalidad (25).

En cuanto a las complicaciones, las únicas reseñables son las escaras faciales, presentes en el $37 \%$ de los casos. Esta cifra es mayor que la de la mayoría de los trabajos publicados donde no superan el 18\% $(12,15,23)$, pero, también en nuestro caso es superior el número de horas de ventilación ininterrumpida.

La estancia hospitalaria media de nuestros pacientes está dentro de la reconocida en otros trabajos, que va desde los 9 a los 16 días $(16,18,19,23)$ con una media de $(9,4 \pm 1,6)$ días (9). Además nuestro estudio se ha realizado sin aumentar la dotación de personal de enfermería en la planta de hospitalización aunque si que se incrementó la carga de trabajo en las primeras horas del inicio del soporte ventilatorio. Este hecho ocurre fundamentalmente durante las primeras 8 horas desde el ingreso $(16,26)$.

En definitiva podemos concluir que la utilización de la VNI en una planta de hospitalización es útil y segura en el tratamiento de pacientes EPOC con fracaso ventilatorio agudo.

\section{AGRADECIMIENTOS}

Al Dr. Bugarín González del Servicio de Urgencias por la ayuda prestada para la realización de este trabajo. tive pressure ventilation in the setting of severe, acute exacerbations of chronic obstructive pulmonary disease: more effective and less expensive. Crit Care Med 2000; 28: 2094-2102.

4. International consensus conferences in intensive care medicine: noninvasive positive pressure ventilation in acute respiratory failure. Am J Respir Crit Care Med 2001; 163: 283-291.

5. Mehta S, Hill NS. Non-invasive ventilation. State of the art. Am J Respir Crit Care Med 2001; 163: 540-577.

6. Pauwels RA, Buist AS, Calverley PM, Jenkins CR, Hurd SS; GOLD 
Scientific Committee. Global strategy for the diagnosis, management, and prevention of chronic obstructive pulmonary disease. NHLBI/WHO Global Initiative for Chronic Obstructive Lung Disease (GOLD) Workshop summary. Am J Respir Crit Care Med 2001; 163: 1256-1276.

7. British Thoracic Society Standards of Care Committee. Non-invasive ventilation in acute respiratory failure. Thorax 2002; 57: 192-211.

8. Marrades RM, Rodríguez Roisin R. Enfermedad pulmonar obstructiva crónica y ventilación no invasiva: una evidencia creciente. Arch Bronconeumol 2001; 37: 88-95.

9. Fernández Guerra J, López-Campos Bodineau JL, Perea-Milla López E, Pons Pellicer J, Rivera Irigoin R, Moreno Arrastio LF. Metanálisis de la eficacia de la ventilación no invasiva en la exacerbación aguda de la enfermedad pulmonar obstructiva crónica. Med Clin (Barc) 2003; 120: 281-286.

10. Benhamou D, Girault C, Faure C, Portier F, Muir JF. Nasal mask ventilation in acute respiratory failure: experience in elderly patients. Chest 1992: 102: 912-917.

11. Vitacca M, Rubini F, Foglio K, Scalvini S, Nava S, Ambrosino N. Non-invasive mechanical ventilation in severe chronic obstructive lung disease and acute respiratory failure: short- and long-term prognosis. Intensive Care Med 1993; 19: 450-455.

12. Kramer N, Meyer TJ, Meharg J, Cece RD, Hill NS. Randomized prospective trial of noninvasive positive pressure ventilation in acute respiratory failure. Am J Respir Crit Care Med 1995; 151: 1799-806.

13. Solsona JF, Miro G, Ferrer M, Cabré L, Torres A. Los criterios de ingreso en la UCI del paciente con enfermedad obstructiva crónica. Documento de Consenso Sociedad Española de Medicina Intensiva Crítica y Unidades Coronarias (SEMICYUC), Sociedad Española de Neumología y Cirugía Torácica (SEPAR). Arch Bronconeumol 2001; 37: 335-339.

14. Plant PK, Owen JL, Parrott S, Elliott MW. Cost effectiveness of ward based non-invasive ventilation for acute exacerbations of chronic obstructive pulmonary disease: economic analysis of randomised controlled trial. BMJ 2003; 326: 1-5.

15. Antón A, Guell R, Gómez J, Serrano J, Castellano A, Carrasco JL, Sanchis J. Predicting the result of noninvasive ventilation in severe acute exacerbations of patients with chronic airflow limitation. Chest 2000; 117: 828-833.

16. Plant PK, Owen JL, Elliott MW. Early use of non-invasive ventilation for acute exacerbations of chronic obstructive pulmonary disease on general respiratory wards: a multicentre randomised controlled trial. Lancet 2000; 355: 1931-1935.

17. Foglio C, Vitacca M, Quadri A, Scalvini S, Marangoni S, Ambrosino N. Acute exacerbations in severe COLD patients. Treatment using positive pressure ventilation by nasal mask. Chest 1992;101: 1533-1538

18. Barbé F, Togores B, Rubi M, Pons S, Maimo A, Agusti AGN. Noninvasive ventilatory support does not facilitate recovery from acute respiratory failure in chronic obstructive pulmonary disease. Eur Respir J 1996; 9: 1240-1245.

19. Bott J, Carroll MP, Conway JH, Keilty SE, Ward EM, Brown AM, Paul EA, Elliott MW, Godfrey RC, Wedzicha JA, Moxham J. Randomised controlled trial of nasal ventilation in acute ventilatory failure due to chronic obstructive airways disease. Lancet 1993; 341: 1555-1557.

20. Jeffrey AA, Warren PM, Flenley DC. Acute hypercapnic respiratory failure in patients with chronic obstructive lung disease: risk factors and use of guidelines for management. Thorax 1992; 47: 34-40.

21. Roberts CM, Lowe D, Bucknall CE, Ryland I, Kelly Y, Pearson MG. Clinical audit indicators of outcome following admission to hospital with acute exacerbation of chronic obstructive pulmonary disease. Tho$\operatorname{rax} 2002 ; 57:$ 137-41.

22. Seneff MG, Wagner DP, Wagner RP, Zimmerman JE, Knaus WA. Hospital and 1-year survival of patients admitted to intensive care units with acute exacerbation of chronic obstructive pulmonary disease. JAMA 1995; 274: 1852-1857.

23. Confalonieri M, Parigi P, Scartabellati A, Aiolfi S, Scorsetti A, Nava S, Gandola L. Noninvasive mechanical ventilation improves the immediate and long-term outcome of COPD patients with acute respiratory failure. Eur Respir J 1996; 9: 422-430.

24. Bardi G, Pierotello R, Desideri M, Valdisserri L, Bottai M, Palla A. Nasal ventilation in COPD exacerbations: early and late results of a prospective, controlled study. Eur Respir J 2000; 15: 98-104.

25. Dueñas-Pareja Y, López-Martín S, García-García J, Melchor R, Rodríguez-Nieto MJ, González-Mangado N, Peces-Barba G. Ventilación no invasiva en pacientes con encefalopatía hipercápnica grave en una sala de hospitalización convencional. Arch Bronconeumol 2002; 38: 372 375.

26. Chevrolet JC, Jolliet P, Abajo B, Toussi A, Louis M. Nasal positive pressure ventilation in patients with acute respiratory failure. Difficult and time- consuming procedure for nurses. Chest 1991; 100: 775-782. 